\title{
Clinical Depression
}

\section{How Emotional Expressivity and Depression Might Explain Somatization in Iranian Clinically Depressed Patients}

\author{
Hossein Kaviani $^{1^{*}}$, Zahra Mirsepassi ${ }^{2}$ and Marzieh Mousavi ${ }^{3}$ \\ ${ }^{1}$ School of Psychology, University of Bedfordshire, Luton, UK \\ ${ }^{2}$ Department of Psychiatry, Rouzbeh Hospital, Tehran University of Medical Sciences, Tehran, Iran \\ ${ }^{3}$ Department of Clinical Psychology, Tehran Medical Sciences Branch, Islamic Azad University, Tehran, Iran
}

*Corresponding author: Hossein Kaviani, School of Psychology, University of Bedfordshire, Luton, UK, Tel: +44-1582743765; E-mail: Hossein.Kaviani@beds.ac.uk

Received date: July 11, 2018; Accepted date: July 25, 2018; Published date: August 02, 2018

Copyright: $\odot 2018$ Kaviani $\mathrm{H}$ et al. This is an open-access article distributed under the terms of the Creative Commons Attribution License, which permits unrestricted use, distribution, and reproduction in any medium, provided the original author and source are credited.

\begin{abstract}
Somatization is characterised by a tendency to experience and present psychological distress through somatic symptoms with no known physical basis. It is a phenomenon that can be found in diverse cultural contexts. A total of 100 Iranian patients with major depressive disorder participated in the present study. A set of self-report questionnaires were used to measure depression, emotional expressivity and somatization. Multiple Regression Analysis yielded that only depression and gender were significant factors to predict somatization. Emotional expressivity did not appear to predict the outcome variable. It is in line with inter-correlations findings that show depression is positively correlated with somatization; no significant correlation was found between emotional expressivity and somatization. Moderation analysis did not confirm any impact of emotional expressivity on the link between depression and somatization. Moreover, gender factor turned out to predict somatization. In the present study, female depressed patients reported higher levels of depression and somatization than their male counterparts. However, they did not differ on emotional expressivity. Some of the present findings could challenge previous research evidence and theoretical accounts; this will be discussed.
\end{abstract}

Keywords: Depression; Emotional expressivity; Somatization; Culture; Iran

\section{Introduction}

It has been always a question of importance whether or not somatic symptoms and signs can be explained by psychological pains and how this might be mediated by culture $[1,2]$. Research evidence suggest that the estimates of somatization prevalence vary from $3 \%$ to $35 \%$ of patients seen in primary health care systems of different cultural, ethnic settings [3-6]. This would point to the fact that somatization is a potentially challenging matter in primary care clinics, since practitioners would find it difficult to decide on differential diagnosis in order to distinguish somatization from purely physical conditions. With this in mind, it can be assumed that the likelihood of misdiagnosis and consequently mal-practice would increase. In fact, insight into various levels of somatization tendency in different cultural, ethnic groups, would help practitioners in primary care to be more successful to avoid such a clinical dilemma.

Evidence suggest that people with Asian cultural background tend to express their psychological pains through somatic symptoms $[7,8]$. Recently, Dreher et al. [9] demonstrate that Vietnamese depressed patients reported higher levels of somatic symptoms than their German counterparts, though both groups turned out to be similar in terms of depression severity. It has been pointed out that 'somatization can be understood as a cultural script for depression'; implying that in some cultural contexts, e.g., Chinese society, people tend to somatise as they may actually experience a set of bodily pains and aches or it may be used as a strategy to avoid stigma in communications [10]. In fact, Ryder et al. [10] conducted confirmatory factor analysis and structural equation modelling concluding that although Chinese somatization could be attributed to these two factors (experience of bodily pains and stigma-avoiding communication strategy), only bodily experience is closely bound up with somatic depressive symptoms.

Moreover, there are studies [11] which concluded that those who score low on introversion (those who are reserved, less talkative, less sociable) are more likely to somatise their emotional vexation and agony. This might be supported by the evidence that suggest tendency to somatise emotional pains can be alleviated when the ability to express feelings is enhanced [12].

The link between emotional expressivity and somatization is further supported in a study [13] on Hispanic patients compared with their White American counterparts at a primary health care level with Hispanics being more likely to report somatic symptoms. The present study aimed to examine the relationship between depression, emotional expressivity and somatization in clinically depressed patients within a new cultural setting, namely, an Iranian sample. We are also interested to know if gender would predict the tendency towards somatization in this particular sample. Although there is no previous evidence to confirm if Iranian culture might play a determining role in the tendency towards somatization, a different line of research, namely, on the language that people use for their communication reveals relevant notions. In keeping with this, a research was carried out to scrutinise Persian vocabularies in terms of their emotional tone embedded in words [14]. The authors reported that Iranians predominantly use body-related words to communicate their emotions. With this in mind, one can assume that people living in a less expressive culture, are not much encouraged to express their feelings and thoughts, therefore tend to somatise their psychological complaints and emotional problems [15-17]. It might imply that clinicians should be conscious about the linguistic context in which the 
symptoms and signs are communicated by patients, which then help them with case conceptualization more effectively.

\section{Method}

\section{Participants and procedure}

A total of 100 depressed patients were recruited from an outpatient clinic, a private office and a psychiatric ward (Rouzbeh Psychiatric Hospital, Tehran) participated in this study. They were interviewed individually by a psychiatrist and a clinical psychologist using DSM-V [18] diagnostic criteria for Major Depressive Disorder (MDD); patients with psychotic features were excluded from the study sample. Table 1 depicts the demographic details of the sample in the two groups. The study followed the BPS code of ethics and participants signed an informed consent form (including a full description of the project) prior to taking part in the study.

\begin{tabular}{|l|l|l|}
\hline \multirow{3}{*}{ Gender } & Male & $33(33 \%)$ \\
\cline { 2 - 3 } & Female & $67(67.7 \%)$ \\
\hline \multirow{4}{*}{ Marital status } & Mean & 34.78 \\
\cline { 2 - 3 } & SD & 10.7 \\
\hline \multirow{3}{*}{ Educational degree } & Single & $32(32 \%)$ \\
\cline { 2 - 3 } & Married & $57(57 \%)$ \\
\cline { 2 - 3 } & $\begin{array}{l}\text { Divorced/widowed/ } \\
\text { separated }\end{array}$ & $11(11 \%)$ \\
\cline { 2 - 3 } & University & $56(56 \%)$ \\
\hline
\end{tabular}

Table 1: Demographic variations of the sample.

\section{Measurements}

Three self-report scales and a demographic questionnaire were employed for data collection on depression, emotional expressivity and somatization. Cronbach's alphas for the scales range from 0.68 to 0.78 which support a satisfactory internal consistency of each of the scales used in this study.

PHQ-9 (Patient Health Questionnaire-9) [19] consists of 9 items relating to depression symptoms. Each item was rated using a 4-point Likert scale ranging from 0 ('Not at all') to 3 ('Nearly every day').

EES (Emotional Expressivity Scale) [20] encompasses 17 items to measure the extent to which people outwardly display their emotions. To rate each item, a 6-point Likert from 1 ('never true of me') to 6 ('generally true of me') was utilized.

PHQ-15T (Patient Health Questionnaire-15) [21] was used to measures level of tendency for somatization. The scale consisted of 15 items with 3-point Likert rating scale from 0 ('Not bothered at all') to 2 ('Bothered a lot').

In addition, a demographic questionnaire was also used to detect details concerning gender, age, marital status and education background.

\section{Data analysis}

The data was statistically analysed using SPSS 22. To examine potential predictive links between independent variable (depression, emotional expressivity, gender and age) with the outcome variable (somatization), a hierarchical multiple regression was used, followed by a moderation analysis to see if emotional expressivity affects the link between depression and somatization. To gauge potential gender differences on different variables, a series of t-tests was conducted. Pearson correlation was performed to examine the variables intercorrelations.

\section{Results}

\section{Demographics}

Participants were more female (two-third) than men. In terms of marital status, majority of the participants were married; one-third was single. Concerning education, the number of participants who held high school degree was slightly higher than those who were either student or graduated.

\section{Predictive link}

Multiple regression analysis: A hierarchical multiple regression analysis was utilised to examine the predictive value of the measures. Demographic variables (age and gender) were entered in the first step and psychological variables (emotional expressivity and depression) in the second step. Table 2 summarizes essential details of the regression analyses for the sample.

\begin{tabular}{|c|c|c|}
\hline \multicolumn{3}{|l|}{ Step 1: Demographics } \\
\hline & Beta & $\mathrm{R} 2$ range \\
\hline Age & $-0.13, \mathrm{~ns}$ & $0.14, p<0.001$ \\
\hline Gender & $0.33, p<0.01$ & \\
\hline \multicolumn{3}{|c|}{ Step 2: Psychological variables } \\
\hline Emotional expressivity & $-0.10, \mathrm{~ns}$ & $0.25, p<0.001$ \\
\hline Depression & $0.52, p<0.001$ & \\
\hline
\end{tabular}

Table 2: Regression analysis results.

The findings reveal demographic variables (age and gender) account for $14 \%$ of the variation in somatization with gender making a significant contribution. Psychological variables (emotional expressivity and depression) explain $25 \%$ of variance in somatization with depression making a significant, particularly large contribution. The moderation analysis showed the link between depression and somatization is not moderated by emotional expressivity.

\section{Inter-correlations}

Pearson correlations between variables show that while depression is positively correlated with somatization, no significant association was obtained between emotional expressivity and somatization in this particular sample. Moreover, a positive correlation was found between depression and emotional expressivity. 


\begin{tabular}{|l|l|l|l|}
\hline & Somatization & Depression & $\begin{array}{l}\text { Emotional } \\
\text { expressivity }\end{array}$ \\
\hline Somatization & 1 & $0.55, \mathrm{p}<001$ & $0.07, \mathrm{~ns}$ \\
\hline Depression & & 1 & $0.21, \mathrm{p}<0.05$ \\
\hline $\begin{array}{l}\text { Emotional } \\
\text { expressivity }\end{array}$ & & & 1 \\
\hline
\end{tabular}

Table 3: Pearson correlation between the study variables.

\section{Gender differences}

To examine potential gender differences, a series of independent $\mathrm{t}$ tests was conducted on the target variables, namely somatization, depression and emotional expressivity. Table 4 shows that women scored more highly on somatization and depression than their male counterparts. Female and male participants did not appear to differ significantly on emotional expressivity (Figure 1).

\begin{tabular}{|l|l|l|l|l|}
\hline Variables & Mean (SD) & Df & t-value & Sig \\
\hline \multirow{2}{*}{ Somatization } & Men 9.27(4.61) & \multirow{2}{*}{98} & 3.97 & $<0.001$ \\
\cline { 2 - 5 } & Women 13.57(5.93) & & & \\
\hline \multirow{2}{*}{ Depression } & Men 13.00 (5.59) & \multirow{2}{*}{98} & 2.2 & $<0.05$ \\
\cline { 2 - 5 } & Women 15.75(6.33) & & ns \\
\hline \multirow{2}{*}{$\begin{array}{l}\text { Emotional } \\
\text { expressivity }\end{array}$} & Men 52.97(14.14) & \multirow{2}{*}{98} & 0.7 & \\
\cline { 2 - 5 } & Women 51.03(10.07) & & & \\
\hline
\end{tabular}

Table 4: T-tests results for gender differences on different variables.

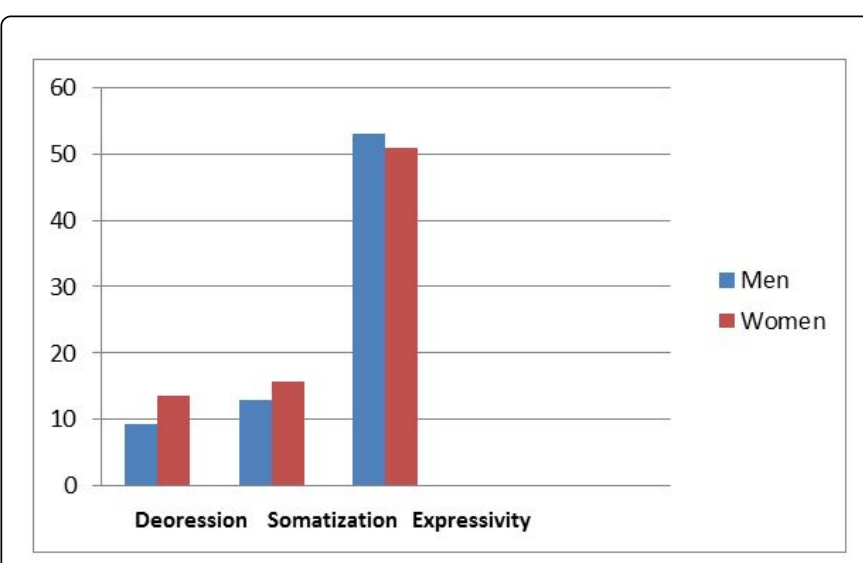

Figure 1: Gender differences on depression, somatization and emotional expressivity.

\section{Discussion}

The main objective in this study was to examine the associations between depression, emotional expressivity and somatization among clinically depressed patients in a new cultural setting, namely Iranian society. In general, the present findings obtained in the present study differ from those in a previous study carried out in Hispanics and White Americans [13]. It will be further discussed and explained later.

Among psychological factors, only depression and gender were found to be the main criterion variables to significantly predict somatization. Emotional expressivity did not appear to predict the outcome variable. Moreover, moderation analysis did not confirm any impact of emotional expressivity on the link between depression and somatization. Inter-correlations between variables demonstrate that although depression is positively correlated with somatization, no significant correlation was found between emotional expressivity and somatization. This further supports the findings of regression and moderation analysis. However, the present result is not consistent with the findings of previous studies $[12,13]$ which suggest that a predictive link exists between emotional expressivity and somatization. It has been previously pointed out that emotional difficulties can be expressed by presenting physical symptoms [15-17]. Besides, it has been postulated that people in Asian countries tend to express their emotional distress through somatic symptoms [7,8]. This notion is not, however, supported by the present findings on Iranian depressed patients. It is in line with the recent evidence that has challenged this hypothesis and indicated that it is not the cultural background, but the adverse life event such as immigration which influences somatization [22]. It is suggested that future studies need to control for recent life adversities as potential confounding variables. Based on the lack of consistency among research findings on somatization, there have been suggestions [23] to de-emphasise somatoform disorders as an independent diagnostic category and even move it from Axis I to Axis II in DSM taxonomy. Somatoform disorders were introduced as a diagnostic entity in DSM III for bodily symptoms and signs not being medically explained. This diagnostic category remained in DSM IV and DSM V, although the heated controversy continued over whether to keep somatoform disorders as an independent category or regard it as auxiliary details in Axis II of other independent categories such as depression.

Among demographic factors, only gender turned out to predict somatization. That is, the only demographic factor contributing to somatization level is gender. Further analysis show that female patients are higher on depression and somatization than men. They did not differ on emotional expressivity. It is in line with previous research findings [13]. There are studies [22,23], reporting that female participants are more likely than their male counterparts to somatise. However, the effect of gender on the expression of somatic symptoms has been challenged by research evidence [24-26].

This research might provide some clinical implications for clinicians in the field of medicine. For example, the overlap between somatoform symptoms (not medically explained) and those of physical ailments may cause a practical dilemma for practitioners particularly for physicians working in primary health care systems. This might lead to misdiagnosis and as a result, malpractice. As suggested by some researchers, it seems crucial to raise awareness for cultural dynamics of the tendency to somatise in depressed patients [9]. Any related guideline or training course to enhance cultural competence among clinicians can be informed by the present findings with the hope that the results as such would help practitioners to tackle this dilemma.

The inferences derived from the findings of present study should be interpreted and generalized with caution owing to its limitations including the nature of sample (clinical) and sampling method (convenience). Future studies need to take a wider sample from both general and clinical populations in various geographical locations. Furthermore, there might be some confounding factors, such as 
adverse life event, level of emotionality and the like that can be recorded and controlled in future research.

\section{References}

1. Busch FN (2014) Clinical approaches to somatization. J Clin Psychol 70: 419-427.

2. Kirmayer LJ, Robbins JM (1991) Three forms of somatization in primary care: prevalence, co-occurrence, and sociodemographic characteristics. J Nerv Ment Dis 179: 647-655.

3. Maany FH (1981) Treatment of depression associated with Briquet's syndrome. Am J Psychiatry 138: 373-376.

4. Escobar JI, Gara M, Silver RC, Waitzkin H, Holman A, et al. (1998) Somatization disorder in primary care. Br J Psychiatry 173: 262-266.

5. Gureje O1, Simon GE, Ustun TB, Goldberg DP (1997) Somatization in cross-cultural perspective: A World Health Organization Study in Primary Care. Am J Psychiatry 154: 989-995.

6. Peveler R, Kilkenny L, Kinmonth AL (1997) Medically unexplained physical symptoms in primary care: a comparison of self-report screening questionnaires and clinical opinion. J Psychosom Res 42: 245-252.

7. Farooq S, Gahir MS, Okyere E, Sheikh AJ, Oyebode F (1995) Somatization: A transcultural study. J Psychosom Res 39: 883-888.

8. Ryder AG, Yang J, Zhu X, Yao S, Yi J, et al. (2008) The Cultural Shaping of Depression: Somatic Symptoms in China, Psychological Symptoms in North America? J Abnorm Psychol 117: 300-313.

9. Dreher A, Hahn E, Diefenbacher A, Nguyen MH, Böge K (2017) Cultural differences in symptom representation for depression and somatization measured by the PHQ between Vietnamese and German psychiatric outpatients. J Psychosom Res 102: 71-77.

10. Zhou X, Peng Y, Zhu X, Yao S, Dere J (2016) From culture to symptom: Testing a structural model of "Chinese somatization". Transcult Psychiatry 53: 3-23.

11. Hyphantis T, Goulia P, Carvalho AF (2013) Personality traits, defense mechanisms and hostility features associated with somatic symptom severity in both health and disease. J Psychosom Res 75: 362-369.

12. Kim S, Ki J (2014) A case study on the effects of the creative art therapy with stretching and walking meditation - Focusing on the improvement of emotional expression and alleviation of somatisation symptoms in a neurasthenic adolescent. The Arts in Psychotherapy 41: 71-78.

13. Kaviani H, Tabrizi MC (2016) Emotional expressivity and somatization symptoms in clinically depressed patients. Clin Depress 3: 113.

14. Kaviani H, Pournaseh M, Sagan O (2015) Emotion-related words in Persian Dictionaries: Culture, meaning and emotion theory. International Journal of Linguistics, Literature and Culture 2: 1-11.
15. King LA, Emmons RA (1991) Psychological, physical and interpersonal correlates of emotional expressiveness, conflict and control. European Journal of Personality, 5: 131-150.

16. Kleinman A, Kleinman J, Good B (1985) Somatization: The Interconnections in Chinese Society among Culture, Depressive Experiences, and the Meanings of Pain. In: Kleinman A and Good B (eds), Culture and Depression: Studies in the Anthropology and CrossCultural Psychiatry of Affect and Disorder. University of California Press, Berkeley, US, pp. 429-490.

17. Pennebaker JW (1995) Emotion, disclosure, and health. American Psychological Association, Washington, DC, USA.

18. American Psychiatric Association (2013) Diagnostic and Statistical Manual of Mental Disorders. (5thedn), American Psychiatric Publishing, Arlington, VA.

19. Löwe B, Kroenke K, Herzog W, Gräfe K (2004) Measuring depression outcome with a brief self-report instrument: sensitivity to change of the Patient Health Questionnaire (PHQ-9). J Affect Disordv81: 61-66.

20. Kring AM, Smith DA, Neale JM (1994) Individual differences in dispositional expressiveness: development and validation of the emotional expressivity scale. J Pers Soc Psychol 66: 934-349.

21. de Vroege L, Hoedeman R, Nuyen J, Sijtsma K, van der Feltz-Cornelis CM (2012) Validation of the PHQ-15 for somatoform disorder in the occupational health care setting. J Occup Rehabil 22: 51-58.

22. Bragazzi NL, Puente GD, Natta WM (2014) Somatic perception, cultural differences and immigration: results from administration of the Modified Somatic Perception Questionnaire (MSPQ) to a sample of immigrants. Psychol Res Behav Manag 7: 161-166.

23. Mayou R, Kirmayer LJ, Simon G, Kroenke K, Sharpe M (2005) Somatoform disorders: Time for a new approach in DSM-V. Am J Psychiatry 162: 847-855.

24. Ali A, Deuri SP, Deuri SK, Jahan M, Singh AR, et al. (2010) Perceived social support and life satisfaction in persons with somatization disorder. Ind Psychiatry J 19: 115-118.

25. Zwaigenbaum L, Szatmari P, Boyle MH, Offord DR (1999) Highly somatizing young adolescents and the risk of depression. Pediatrics 103: 1203-1209.

26. Castro Y, Carbonell JL, Anestis JC (2011) The influence of gender role on the prediction of antisocial behaviour and somatization. Int J Soc Psychiatry 58: 409-416. 